\title{
Probabilistic Compositional Active Basis Models for Robust Pattern Recognition
}

\author{
Adam Kortylewski \\ adam.kortylewski@unibas.ch \\ Thomas Vetter \\ thomas.vetter@unibas.ch
}

\author{
Department of Mathematics and Computer \\ Science \\ University of Basel \\ Basel, Switzerland
}

In this paper we propose an approach for learning hierarchical compositional active basis models. Our contribution is three-fold: First, we introduce a greedy EM-type algorithm to automatically infer the complete structure of a compositional active basis model (CABM). Second, we formulate the pattern model and the learning process in a fully probabilistic manner. Finally, based on the statistical framework, we augment the pattern model with an implicit geometric background model that reduces the models sensitivity to pattern occlusions and structured clutter. We demonstrate that probabilistic CABMs are capable of recognising patterns under complex non-linear distortions that can hardly be represented by a finite set of training data.

Probabilistic hierarchical compositional models have been proposed as object representation e.g. in $[1,2,3]$. However, in contrast to [1], we automatically learn the structure of the hierarchy. The work in $[2,3]$ is most related to our method. In difference to [3] we embed our model in a statistical inference framework. Compared to [2], we use fully generative compositional units instead of invariant features as part representa- tions. Furthermore, we do not make hard decisions on the detection of parts during learning. Instead the full part likelihoods are used in the structure induction process.

In order to demonstrate the robustness of the proposed object representation, we evaluate it on a complex forensic image analysis task (Fig.1). We learn CABMs for 1175 reference impressions. Subsequently, the posterior probability of 300 probe images under each reference model is inferred within a Bayesian estimation setup. Experimental results show that the forensic image analysis task is processed with unprecedented quality.

[1] Jifeng Dai et al. Unsupervised learning of dictionaries of hierarchical compositional models. In CVPR, 2014.

[2] Long Zhu et al. Unsupervised structure learning: Hierarchical recursive composition, suspicious coincidence and competitive exclusion. In ECCV, 2008.

[3] Sanja Fidler and Aleš Leonardis. Towards scalable representations of object categories: Learning a hierarchy of parts. In $C V P R, 2007$.

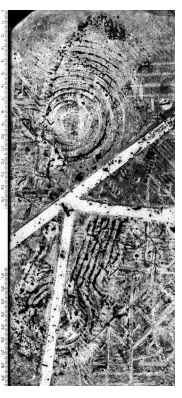

(a)

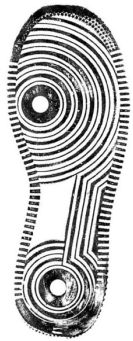

(b)
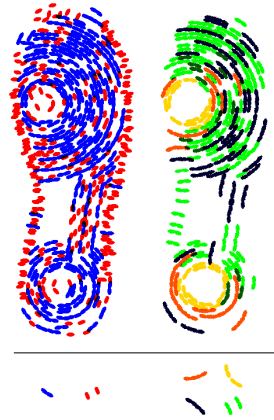

(d)
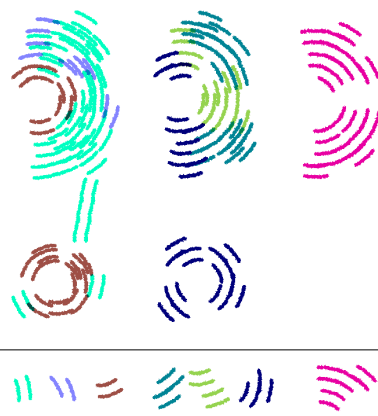

(e)

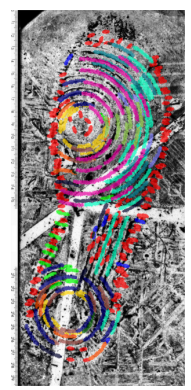

(h)

Figure 1: Overview over the process of footwear impression recognition. (a) A typical probe image. (b) The corresponding reference impression; (c-g) The learning result for each layer of the compositional hierarchy. The learned CABMs are illustrated with different colours in their mean position (bottom), together with an encoding of reference impression (top). (h) An overlay of the final CABM over the probe image with the spatial transformation that maximises the posterior probability. 